\title{
«Casser la branche militaire »: Claudel et la poétique de fin de siècle
}

\author{
Sergio Villani \\ Université York
}

Celui-là seul saura sourire, s’il a plu

A la Muse elle-même, institutrice et Mère,

De former, lui ouvrant la Lettre et la Grammaire,

Sa lèvre au vers exact et au Mot absolu.

La sécurité de l'office qui l'élut

Rit que rien d'éternel comme rien d'éphémère

N'échappe à la mesure adéquate et sommaire

De la voix qui finit ou le verbe conclut.

Gardien pur d'un or fixe où l'aboi vague insulte!

$\mathrm{Si}$, hommage rustique et témoignage occulte,

Ma main cherche quoi prendre au sol pour s'en armer,

Je choisis de casser la branche militaire

Dont la feuille à ton front honore, Mallarmé,

Amère, le triomphe, et verte, le mystère.

C

e sonnet, « Celui-là saura sourire..., » inclut à la fin des « Premiers vers, » $(\mathrm{OP}, 10)^{1}$ est écrit à Han-Kéou en juillet 1897 pour un recueil d'hommages offerts à Stéphane Mallarmé (Voir OP, 1O21). L'initiative est celle d'Albert Mockel² et les participants, une vingtaine de jeunes disciples du cénacle mallarméen qui se réunissent le mardi soir à la rue de Rome, chez le Poète. Claudel, à vrai dire, n'avait assisté que trois ou quatre fois à ces augustes assemblées de 1887 à 1890, mais il avait gardé le contact avec Mallarmé3 de 1893 à 1897, au cours de ses séjours diplomatiques aux États-Unis et en Chine. Le sonnet est un hommage à la figure du poète incarnée par Mallarmé et une illustration imitative, virtuose, de sa pratique de poésie. Cependant, ce poème représente aussi un point de démarcation, une séparation de cette " école » symboliste de fin de siècle dont Mallarmé a été consacré le Prince des Poètes. Cette distanciation du jeune Claudel s'opère à travers une dialectique apprise de ses 
lectures d'Aristote et de saint Thomas4 et surtout par l'emploi de la « loi de l'analogie ", apprise de saint Bonaventure5, comme principe fondamental d'expression poétique. Comme Yves Bonnefoy écrira dans les années 50 qu'il " faut oublier Valéry ", afin de trouver une nouvelle voix/voie poétique, Claudel déclare la nécessité de "casser " avec la vision mallarméenne et sa pratique de poésie qui n'aboutissent qu'au " rien ", à l'absence et l'imitation stérile.

Plusieurs critiques ont essayé de relier l'ascendance littéraire de Claudel à Mallarmé6 bien que lui, dès le début, se soit inscrit dans la lignée de Rimbaud. Déjà, dans la lettre qui accompagne l'envoi de son sonnet-hommage à Mallarmé, il affirme y avoir, entre lui et Rimbaud, ce qu'il nomme « un si intime hymen de deux esprits » (Correspondance Mallarmé-Claudel, 54) En fait, tout le long de sa vie - même le vieux poète -, prendra ses distances de Mallarmé et des poètes de sa souche, et surtout de Paul Valéry. Il considère Mallarmé un suprême versificateur; il l'appelle « un technicien », « un analyste », « un théoricien », $(M I, 66)$, la plus parfaite expression de l'idéal symboliste. Pourtant, convaincu que la forme, n'importe laquelle, doit toujours servir l'expression ou s'y adapter, il reproche à Mallarmé d'avoir fait l'inverse, et surtout de ne pas s'être libéré de ce qu'il appelle « l'accoutrement arithmétique de l'alexandrin » $(M I, 44)$ et d'autres contraintes formelles. Bref, Mallarmé est pour lui un autre rhétoriqueur, même s'il est grand dans ce domaine.

Les divergences au niveau idéologique sont plus extrêmes. Déjà en 1897, Claudel écrit à Mallarmé qu'il ne partage pas ses opinions sur Rimbaud et sur le catholicisme, telles que proférées dans deux écrits récents de Mallarmé. L'opposition est fondamentale. Les deux poètes poursuivent un idéal : Mallarmé le "Grand Livre ", Claudel cette « Totalité » cosmique. Mais tandis que celui-ci, Claudel, atteint cette « conaissance ", perçoit ce que Baudelaire appelle une profonde unité, même si ténébreuse parfois; la tentative de Mallarmé n'aboutit qu'à l'échec, à la constatation de la fragmentation, du trou noir, de l'absence. Claudel sera toujours reconnaissant d'avoir appris de Mallarmé à toujours chercher la signification, à se poser toujours la question : qu'est-ce que cela veut dire? ( $M I, 65)$. Lui, il trouve une réponse signifiante à ces interrogations ; l'autre, Mallarmé, ne trouve que le silence, car Mallarmé, d'après Claudel, s'est détourné enfin « des recherches spirituelles ou de la langue » $(M I, 66)$.

Cette perte de dimension spirituelle Claudel l'attribue à l'existence urbaine de Mallarmé. La Ville, comme dans son premier drame, exerce une influence corruptrice. Comme les villes bibliques de Sodome 
et Gomorrhe, Claudel voit le Paris des années 80 dans une stagnation spirituelle, dans un bagne de matérialisme. Mallarmé, tout comme ses disciples, est pour lui le poète de la ville, celui qui a subi la contamination urbaine. Chaque fois, en fait, qu'il fait référence à Mallarmé, ce sera toujours par rapport à Paris ; la ville lui donne identité. Lui, par contre, se représente, d'une manière apologétique, presque invariablement comme le campagnard, le rustique, celui qui ne connait pas les raffinements de la vie urbaine et le comportement et goûts décadents qui mènent à la déchéance spirituelle et attirent l'anathème divin et la destruction.

Le sonnet-hommage ci-dessus, par sa forme et son fond, célèbre Mallarmé et les valeurs poétiques qu'il représente, mais aussi marque un tournant définitif de Claudel, une distanciation voulue, choisie, d'une poétique qu'il estime trop contraignante et d'une pensée esthétique et existentielle qu'il considère une voie à la perdition. Le sonnet esquisse deux figures de poète qui se rencontrent, comme deux galaxies, se nourrissent de leurs énergies, et puis s'éloignent l'un de l'autre dans leur propre sphère de l'univers pour affirmer leur identité.

Comme dans un récit mythique ou dans un poème épique, les deux premiers quatrains tracent une sorte de généalogie du poète-hérosMallarmé. Dans le premier quatrain, l'évocation de la Muse de la poésie rappelle l'ouverture des Cinq Grandes Odes et l'invocation dans ce poème aux protectrices des arts, aux pouvoirs de leur office. D'abord, il y a référence à la naissance du poète. Le héros-poète a une ascendance divine : il est le fils de la Muse, sa mère-nourrice. Puis il y a référence à la formation du héros. Cette mère/muse est aussi son institutrice; c'est elle qui lui confère la connaissance de la métrique et du langage, la valeur de l'exactitude ( "vers exact ») et l’idéal de l'absolu ( Mot absolu »). Dans Mémoires improvisés (128), Claudel remarque que Mallarmé « s'exprime avec exactitude et précision ". À cause de cette naissance hors du commun et de cette formation particulière dans les arts du langage, l'enfant-poète baigne dans une béatitude exquise, où il sourit comme un petit dieu. Le Mot « absolu » à la fin du premier quatrain rappelle l'expression à valeur superlative et déitique, « Celui-là seul saura sourire... » du premier vers et relie le premier quatrain au deuxième en faisant écho harmoniquement au mot « élut » à la fin du premier vers, pour exprimer l'idée d'une destinée unique.

Le deuxième quatrain continue cette exposition, comme dans un récit épique, en faisant référence aux exploits du poète-héros-élu. La pratique de son art lui a permis de réaliser une expression " sommaire ", 
totalisante, qui réconcilie les extrêmes éternel-éphémère. Rien n'y échappe. La maîtrise de son art et ses accomplissements sont la cause de la satisfaction, du sens de " sécurité » chez "l'office qui l'élut ». Cette complaisance se reconnaît dans le rire de la Muse/mère qui répond au " sourire » de son enfant. L'exposé existentiel de la vie du poète-héros s'achève par la répétition de l'idée de finitude dans les mots « sommaire », « finit » et « conclut » et le résumé de la vie du poète se fait aussi par le moyen d'une image-symbole dans le premier vers du premier tercet, - une image qui établit un lien entre les quatrains et les tercets :

Gardien pur d'un or fixe où l'aboi vague insulte!

Ce vers constitue un toast exclamatif en l'honneur du poète Mallarmé. Le transfert de l'adjectif « pur » de sa place naturelle, près du mot « or », surprend mais, associé au mot " Gardien ", suggère que c'est le poète qui garantit la fixité de la valeur absolue de son produit, l'or-poésie. L'image du poète-gardien renforce et se relie à l'idée de " sécurité » dans le premier vers du deuxième quatrain. Cette image évoque, d'ailleurs, par la voie de l'analogie, un chien de garde virtuel qui donne l'alerte à un danger inexistant. Il n'y a rien à craindre car Mallarmé est un grand défenseur de la poésie.

Cette image du poète-gardien, défenseur absolu de la valeur de la poésie est accompagnée d'une illustration/imitation de son art. Claudel se révèle donc un imitateur virtuose. Il veut démontrer qu'il a maîtrisé complètement le style mallarméen et donc suggérer qu'il faut abandonner cette imitation, s'en séparer, afin de pouvoir poursuivre d'autres voies créatrices, de nouvelles formes adaptées à des expressions individuelles et variées.

L'alexandrin dans ses mains devient malléable, souple, épouse sa voix à toutes les ressources de la rhétorique et aux modulations harmoniques. On a l'impression qu'il se plaît à réaliser de jeux admirables de montreur/prestidigitateur langagier. Notons, par exemple dans le premier quatrain, la répartition équilibrée des mots paires, Muse-Mère, Lettre-Grammaire, exact-absolu. Ce jeu de structures binaires s'amplifie dans le deuxième quatrain, renforcé ici soit par l'équivalence soit par le contraste de sens : rien-rien; éternel-éphémère; adéquate-sommaire; voix-verbe; finit-conclut. Enfin les exemples de ce genre de rhétorique, de "doublets ", ne manquent pas dans les tercets : hommagetémoignage ; rustique-occulte ; amère-verte ; triomphe-mystère. Notons 
aussi, l'ouverture du premier quatrain par une phrase hypothétique, principale-subordonnée, qui est équilibrée dans le premier tercet par une autre phrase hypothétique cette fois-ci avec une structure renversée, subordonnée-principale, structure « chiasmatique», par rapport à l'autre.

Les structures harmoniques exemplifient une pareille dextérité d'imitateur virtuouse. Ces jeux harmoniques sont nombreux. Notons, par exemple, la suite alliterative en « $\mathbf{s}$ »: « Celui-là seul saura sourire », renforcée par les modulations de liquides et labiales $(1, \mathrm{~m}) »$. Lire/Dire ces mots à haute voix et les lèvres ébauchent un sourire; on ne peut que sourire, comme le dieu-poète et sa mère Muse. L'emploi de l'allitération sert aussi à souligner les structures binaires relevées ci-dessus : MuseMère; rien-rien; voix-verbe; feuille-front.

La rime dans ce sonnet offre encore d'exemples intéressants de cette habilité imitatrice de Claudel. Le schéma de la rime miroite la structure de la rime dans de nombreux sonnets de Mallarmé : rime embrassée, avec alternance masculine et féminine, dans les quatrains ; et dans les tercets, distique suivi, encore avec alternance masculine et féminine, d'une rime croisée. Cette rime croisée des tercets, comme les deux parties de la phrase hypothétique (subordonnée-principale) ici, a la fonction de relier ensemble les deux tercets. La rime sert aussi à renforcer certaines idées ou thèmes. La désinence du mot "Grammaire " apparente ce mot à « Mère » et donc suggère que, comme la Muse, la Grammaire et la Lettre ont une responsabilité formatrice dans la vie du poète. Les désinences des mots « éphémère », « sommaire » et « amère », aussi bien que la rime « militaire-mystère », rimes externes et internes, donc, reprennent ce motif harmonique généré par le mot-matrice, Mère. Aussi pourrait-on dire que le mot « taire », dans la rime « militaire-mystère », reprend le thème du silence suggéré dans le deuxième quatrain, « voit qui finit où le verbe conclut ». Claudel, voudra-t-il suggérer aux disciples de Mallarmé qu'il est temps de se taire?... Cet hommage « occulte » cacherait-il donc une intention occulte?

Cette intention / ce message se lit surtout dans la deuxième figure de poète. Celle-ci s'esquisse dans les tercets et forme un pôle opposé, un contrepoint, à la figure mallarméenne des quatrains. La figure du poète dans les tercets, un auto-portrait de Claudel, souligne tout d'abord ses origines « rustiques ». Il n'appartient pas à la ville, ce lieu de corruption, comme Mallarmé. Lui, Claudel, il manifeste ici une attitude belliqueuse, de destructeur. Le "Je choisis " au début du deuxième tercet déclame chez lui une volonté de puissance et d'indépendance. Il n'est 


\section{Sergio Villani}

pas soumis; il refuse de se soumettre à la discipline « militaire », la technique poétique, qui fait marcher le cortège d'imitateurs de Mallarmé. L'image de la branche exprime par le moyen de l'analogie cette intention critique et destructrice de Claudel. La branche est celle du laurier, celle de l'arbre fruitier et celle aussi d'une armée. La Branche vigoureuse, mystérieusement " verte ", - symbole des nombreux imitateurs - qui ceint le front de Mallarmé comme un laurier de vainqueur et de grand poète, lui donnent un triomphe " amer ", car il reconnait la stérilité de l'imitation " militaire ». Cette " branche militaire " est comme une branche gourmande, celle dont le développement épuise la branche à fruits (Le Robert), la production de l'arbre. Pour empêcher cet épuisement, il est nécessaire de la couper. Le verbe " casser » exprime la violence de cette action radicale; il ne faut pas seulement la sevrer, mais la détruire, comme une Ville de pécheurs, comme Sodome et Gomorrhe (cf. la destruction de la Ville dans la pièce de théâtre de 1883, La Ville) . C'est lui, Claudel, qui va le faire avec les armes mêmes de cette armée d'imitateurs car, ironise-t-il par un subtil jeu de mots, Mallarmé est mal armé pour le faire lui même.

Il est nécessaire, donc, de lire ce sonnet dans le contexte des controverses esthétiques de la fin de siècle et dans le contexte du développement spirituel et littéraire de Claudel. Dans cette perspective, ce sonnet est à la fois un hommage sincère à la grandeur de Mallarmé comme un « Gardien pur » du don poétique qu'il a reçu et une critique passionnée - haineuse presque, comme le révèlent les images de la guerre et de l'horticulture - de son style et de sa pensée. Claudel refuse de s'attacher à cette " branche militaire »; il « choisit » de l'attaquer et de détruire cette branche gourmande. On y reconnaît la volonté et la disponibilité qui préparent chez lui l'ouverture à un souffle plus ample, à la gestation des Cinq Grandes Odes.

\section{Notes}

1 Paul Claudel, « Premiers vers », in Euvre poétique. Introduction Stanislas Fumet. Textes établis et annotés par Jacques Petit, ( Paris : Gallimard, Bibliothèque de la Pléiade, 1967). Ce sonnet a été publié pour la première fois par Henri Mondor dans sa Vie de Mallarmé (Gallimard, 1942).

2 Poète et grand admirateur de Mallarmé. Voir Esthétique du symbolisme : Propos de littérature (1894); Stéphane Mallarmé (1899); textes divers. 
Claudel et la poétique de fin de siècle

Précédés d'une étude sur Albert Mockel par Michel Otten, (Bruxelles : Palais des Académies, 1962).

3 Voir Henri Mondor, "Introduction à la Correspondance Claudel-Mallarmé », dans Cahiers Paul Claudel I. "Tête d'Or » et les débuts littéraires. (Paris : Gallimard, 1959), 15-39. Et Stéphane Mallarmé et Paul Claudel, "Correspondance commentée par Henri Mondor », dans Cahiers Paul Claudel I, 40-55.

4 Voir Paul Claudel, Mémoires improvisés. Recueillies par Jean Amourche, (Paris : Gallimard, 1954), 128-129.

5 Ibid., 129-131.

6 Voir, par exemple, Louis Chaigne, Vie de Paul Claudel et genèse de son ceuvre (Tours : Maison Mame, 1961), 53-55; Henri Mondor, «Introduction à la Correspondance Claudel-Mallarmé », op. cit. ; Gilbert Gadoffre, " La filiation mallarméenne de Paul Claudel, » dans Entretiens sur Paul Claudel. Sous la direction de Georges Cattaui et Jacques Madaule, (Paris : Mouton, 1968), 33-. Wallace Fowlie, Mallarmé (Chicago : University of Chicago Press, 1953), p. 273-75. 